Memorias del VII Encuentro Nacional de Experiencias en la Enseñanza de la Biología y la Educación Ambiental y II Congreso Nacional de Investigación en la Enseñanza de la Biología

\title{
ELABORACIÓN E IMPLEMENTACIÓN DE UN SEMINARIO DE DIDÁCTICA DE LA BIOLOGÍA Y SU INTEGRACIÓN CON LAS TIC.
}

\section{DESIGN AND IMPLEMENTATION OF A BIOLOGY DIDACTIC'S SEMINAR AND ITS INTEGRATION WITH ICT.}

\author{
Maria Charrier Melillán ${ }^{1}$ \\ M. A. Rognone ${ }^{2}$
}

\section{Resumen}

La incorporación de las TIC a la educación abrió grandes posibilidades para mejorar los procesos de enseñanza y aprendizaje. No obstante, no alcanza con dotar a las escuelas de computadoras, hace falta un cambio en la organización de la misma y en las competencias digitales de los profesores. El presente seminario tuvo como objetivo que los profesores comprendan que el uso de los recursos didácticos combinados con las TIC promueven un cambio tanto conceptual como metodológico de la enseñanza de las ciencias el que deberá plasmarse en una actividad concreta de enseñanza.

Palabras claves: Didáctica, Biología, TIC.

\section{Abstract}

ICT in education opened up great possibilities for improving teaching and learning processes. However, it is not enough to equip the schools with computers, for which a real change in the organization of digital competencies of both, teachers and schools is needed. The aim of this seminar was to make clear to teachers that teaching strategies combined with ICTs, promote both a conceptual and methodological change in science education, which must be reflected in teaching a particular activity.

Key words: didactics, Biology, ICT.

\section{Introducción}

La incorporación de las tecnologías de la información y la comunicación (TIC) a la escuela, produjo un cambio tanto en la forma de enseñar y aprender conocimientos como en el modo de acceder a ellos. Esta incorporación planteó la necesidad de integrarlas al currículo lo que estimuló a numerosos autores a señalar la importancia de definir ciertos requerimientos para lograr la integración. En tal sentido, Sánchez (2002) recopila la información destacando que: 1. es necesario asumir un cambio de rol del profesor y del alumno, 2. el currículo oriente el uso de las TIC y no a la inversa, 3. se haga un uso invisible de las TIC para hacer visible el aprender, 4 . se produzca un cambio desde una

\footnotetext{
${ }^{1}$ Universidad Nacional de Mar del Plata. Argentina. melillan@mdp.edu.ar

${ }^{2}$ Universidad Nacional de Mar del Plata. Argentina. granderica@gmail.com
} 
Edición Extra-Ordinaria. ISSN 2027-1034 P. p 895 - 902

Memorias del VII Encuentro Nacional de Experiencias en la Enseñanza de la Biologia y la Educación Ambiental y II Congreso Nacional de Investigación en la Enseñanza de la Biología

concepción centrada en las TIC a una concepción centrada en el aprender con las TIC y 5. las habilidades requeridas en el uso de las TIC estén directamente relacionadas con el contenido, la clase y esencialmente unidas a un modelo de aprender lógico y sistemático. Para Choque (2008), la posibilidad de llegar a una verdadera integración, radica en que consideremos a las TIC como una herramienta que ayudará a los estudiantes y a los profesores a acceder a variados recursos para adquirir el conocimiento, a trabajar colaborativamente con sus compañeros, a consultar con especialistas o colegas, a compartir conocimientos y a resolver problemas complejos utilizando herramientas cognitivas. Las TIC ofrecen novedosas herramientas para representar el conocimiento por medio de textos, imágenes, gráficos y videos.

En el año 2010 se crea en Argentina "Conectar igualdad" un programa de inclusión digital, educativa y social cuya finalidad fue proporcionar una computadora a cada estudiante y docente de las Escuelas Secundarias, Escuelas de Educación Especial e Institutos de Formación Docente de todo el país pertenecientes al sector estatal. Así como a capacitar a los docentes en el uso de dicha herramienta y a elaborar propuestas educativas con el objeto de favorecer la incorporación de las mismas en los procesos de enseñanza y aprendizaje (Decreto 459/2010). Este programa cuyo propósito, entre muchos otros, es promover la igualdad de oportunidades a todos los jóvenes del país proporcionando un instrumento que, como ya mencionáramos, achique la brecha digital, educativa y social, ha entregado hasta el presente algo más de 3.000.000 Netbooks. Con vistas a mejorar los procesos de enseñanza y aprendizaje a través de la modificación de las formas de trabajo en el aula y en la escuela y a promover el fortalecimiento de la formación de los docentes para el aprovechamiento de las TIC en el aula, es que se implementaron seminarios de perfeccionamiento de profesores en formación inicial y en activo tanto desde el punto de vista del contenido científico como de las didácticas específicas. Es en este contexto que se elaboró una propuestas de integración de las TIC desde una concepción constructivista de la enseñanza de las ciencias que de respuesta al reto que este nuevo paradigma tecnológico nos impone.

El objetivo general del seminario fue comprender que el uso de los recursos didácticos combinados con las TIC promueven un cambio tanto conceptual como metodológico de la enseñanza de las ciencias.

\section{Los objetivos específicos fueron:}

1. Conocer los fundamentos teóricos que sustentan la aplicación de distintas estrategias o recursos que provienen de la Didáctica de la Biología en el aula. 2. Conocer diversas herramientas tecnológicas que nos brinda Internet. 3. Desarrollar distintas actividades para aplicar las herramientas a situaciones concretas de aprendizaje. 4. Participar en distintos foros a fin de reflexionar e intercambiar opiniones y experiencias entre pares y con las tutoras y 5 . Integrar las estrategias y las herramientas a una situación concreta de enseñanza. 
Memorias del VII Encuentro Nacional de Experiencias en la Enseñanza de la Biología y la Educación Ambiental y II Congreso Nacional de Investigación en la Enseñanza de la Biología

\section{Metodología}

El seminario tuvo como destinatarios a Profesores de Biología de los Institutos de Formación Docente argentinos en formación y en activo. Los cursantes debían cumplir con los siguientes requisitos: acceso a internet, cuenta de correo electrónico activa, ingresar en el campus virtual al menos dos veces por semana, participar activamente en los foros y realizar todas las actividades propuestas.

El seminario se realizó con el auspicio del Instituto Superior de Formación Docente (INFD) cuyo personal administró la plataforma e-ducativa. Este fue completamente virtual y asincrónico y se desarrolló en cuatro etapas:

Primera etapa: planificación general, selección y distribución de los contenidos en las ocho semanas que duraría el curso. Se elaboraron distintos documentos: bienvenida a todos los participantes, presentación de las coordinadoras y de las tutoras en cada una de las aulas y presentación del seminario.

Segunda etapa: desarrollo de los contenidos para cada una de las clases, diseño de las actividades y selección de herramientas y enlaces. Foros de apoyo técnico, comentarios generales, seguimiento de los cursantes y de cierre. Se suministraron lecturas complementarias a las clases, videos, enlaces $y$ archivos en pdf.

Tercera etapa: ejecución del seminario, acompañamiento a las tutoras y seguimiento de los cursantes en relación con su participación en los distintos foros.

Cuarta etapa: cierre del seminario. Mediando la finalización del curso, se solicitó cumplimentar voluntariamente una encuesta de opinión con ítems de respuestas cerradas y abiertas.

Los contenidos seleccionados para la elaboración del seminario fueron:

- El conocimiento profesional y el conocimiento didáctico del contenido. EI conocimiento profesional: características y saberes que lo integran. El Conocimiento Didáctico del Contenido (CDC): características. La incorporación de los nuevos conocimientos con posterioridad a los propuestos por Lee Shulman.

- Los mapas conceptuales: Origen, marco teórico y ámbitos de aplicabilidad. Un recurso para la enseñanza y el aprendizaje de las ciencias. 
Edición Extra-Ordinaria. ISSN 2027-1034 P. p 895 - 902

Memorias del VII Encuentro Nacional de Experiencias en la Enseñanza de la Biología y la Educación Ambiental y II Congreso Nacional de Investigación en la Enseñanza de la Biología

- La V de Gowin: Origen, marco teórico y ámbitos de aplicabilidad. Su implementación como recurso para la resolución de problemas, trabajos de laboratorio y de campo y la Historia de la Ciencia.

- La Historia de la Ciencia: Distintas posibilidades y momentos de aplicación como recurso didáctico y su utilización para interpretar la producción de las teorías y del conocimiento científico.

- La resolución de problemas: problemas versus ejercicios. Los problemas como un recurso para generar un cambio conceptual y metodológico de la enseñanza de las ciencias.

Cuando se seleccionaron los contenidos se optó por motivar a los colegas a reflexionar sobre la compleja tarea de dar clase que nos exige contar con unos conocimientos específicos que integran la teoría y la práctica. Estos conocimientos que tenemos los profesores tienen al menos tres características: es profesional, práctico y contextualizado. De allí entonces la incorporación del conocimiento profesional y el CDC. Como el seminario apuntaba a temas referidos a la didáctica de la biología, seleccionamos cuatro estrategias que consideramos importantes para la enseñanza y el aprendizaje de las ciencias naturales. Quedaron pendientes otras cuestiones tanto o más relevantes: trabajos prácticos, salidas de campo, concepciones alternativas, evaluación, otras, que por una cuestión de tiempo no pudimos abordar.

Acompañando el desarrollo de los contenidos propuestos, se utilizaron variadas herramientas y numerosos sitios que complementaban y suplementaban el tratamiento de los mismos. Entre ellos:

- WORDLE: es una aplicación en línea gratuita que permite generar una nube de palabras a partir de un texto o lista de palabras. Se puede modificar con diferentes fuentes, colores y diseños. Se aplicó en: 1. el conocimiento profesional y 2 . para las concepciones alternativas de los cursantes sobre la resolución de problemas en el aula. Permitió exponer y socializar las nubes de forma rápida, atractiva y sencilla de interpretar.

- JING: es un programa gratuito, fácil de instalar y utilizar. Permite capturar imágenes y/o realizar videos seleccionando una zona de la pantalla. Se lo utilizó para capturar la nube de palabra, para la $\mathrm{V}$ de Gowin, otros.

- $\quad$ CMAPTOOLS: es un programa que permite la construcción de mapas conceptuales, fue elaborado por Novak y Cañas como herramienta para la enseñanza, el aprendizaje y la investigación del aprendizaje. Permite de manera sencilla publicar mapas haciéndolos accesibles a otros usuarios. En tal sentido, los profesores pueden colaborar de forma sincrónica o asincrónica en la construcción de los mapas con sus alumnos u otros colegas. Se utilizó para que los 
Edición Extra-Ordinaria. ISSN 2027-1034 P.p 895 - 902

Memorias del VII Encuentro Nacional de Experiencias en la Enseñanza de la Biologia y la Educación Ambiental y II Congreso Nacional de Investigación en la Enseñanza de la Biología

cursantes desplegaran y socializaran las características del CDC y de la fotosíntesis en la actividad de integración.

- $\quad$ Portal educativo chileno: este cuenta con abundante información referida a los elementos que forman la V de Gowin, cómo presentarla a los estudiantes, cómo otorgarle puntaje, ejemplos, otros. Dado la inexistencia de un programa específico, se les aportó una plantilla que los cursantes pudieron usar y guardar para aplicación personal.

- $\quad$ History.com: se utilizaron una serie de videos de la colección del History channel para Historia de la Ciencia.

- Webquest y Miniquest: se utilizaron para la resolución de problemas. Para ello se les proporcionaron numerosos enlaces de cómo utilizar esta herramienta así como ejemplos de variados temas.

Todas las actividades fueron obligatorias. Se elaboraron dos encuestas: 1. Inicial diagnóstica destinada a conocer el perfil de los cursantes y su relación con las TIC y 2. Final de tipo evaluativa donde debían responder sobre los recursos didácticos y las herramientas utilizadas durante el seminario.

Para la evaluación se tuvo en cuenta: la participación en los foros, la realización de las actividades y la presentación de un trabajo final. Este consistió en la elaboración individual de una propuesta docente para la enseñanza y el aprendizaje de un contenido de Biología (a elección del profesor) en la que debían aplicar al menos dos de los recursos vistos. Para la acreditación del seminario debieron tener el $75 \%$ de las actividades aprobadas. El curso no otorgaba puntaje docente.

\section{Resultados y Discusión}

Al seminario se inscribieron un total de 231 docentes en formación inicial y en activo oriundos de 17 provincias y de la Ciudad Autónoma de Buenos Aires los que fueron distribuidos en cuatro aulas, cada uno con una tutora. Cerca del $80 \%$ era de sexo femenino, coincidiendo con la frecuencia de género encontrada habitualmente en el ámbito docente. Del total de inscriptos, 113 completaron la encuesta inicial, a partir de la cual pudimos establecer que los profesores contaban con un mínimo de 1 (un) año y un máximo 40 (cuarenta) años de antigüedad en la docencia.

En relación con la utilización de las TIC con sus estudiantes el $49 \%$ indicó que sí la utilizan, nombrando como principal herramienta el Power Point y en segundo lugar el correo electrónico y/o videos (Figura 1). El 51\% restante no usaba las TIC con los estudiantes. 
Edición Extra-Ordinaria. ISSN 2027-1034 P.p 895 - 902

Memorias del VII Encuentro Nacional de Experiencias en la Enseñanza de la Biologia y la Educación Ambiental y II Congreso Nacional de Investigación en la Enseñanza de la Biología

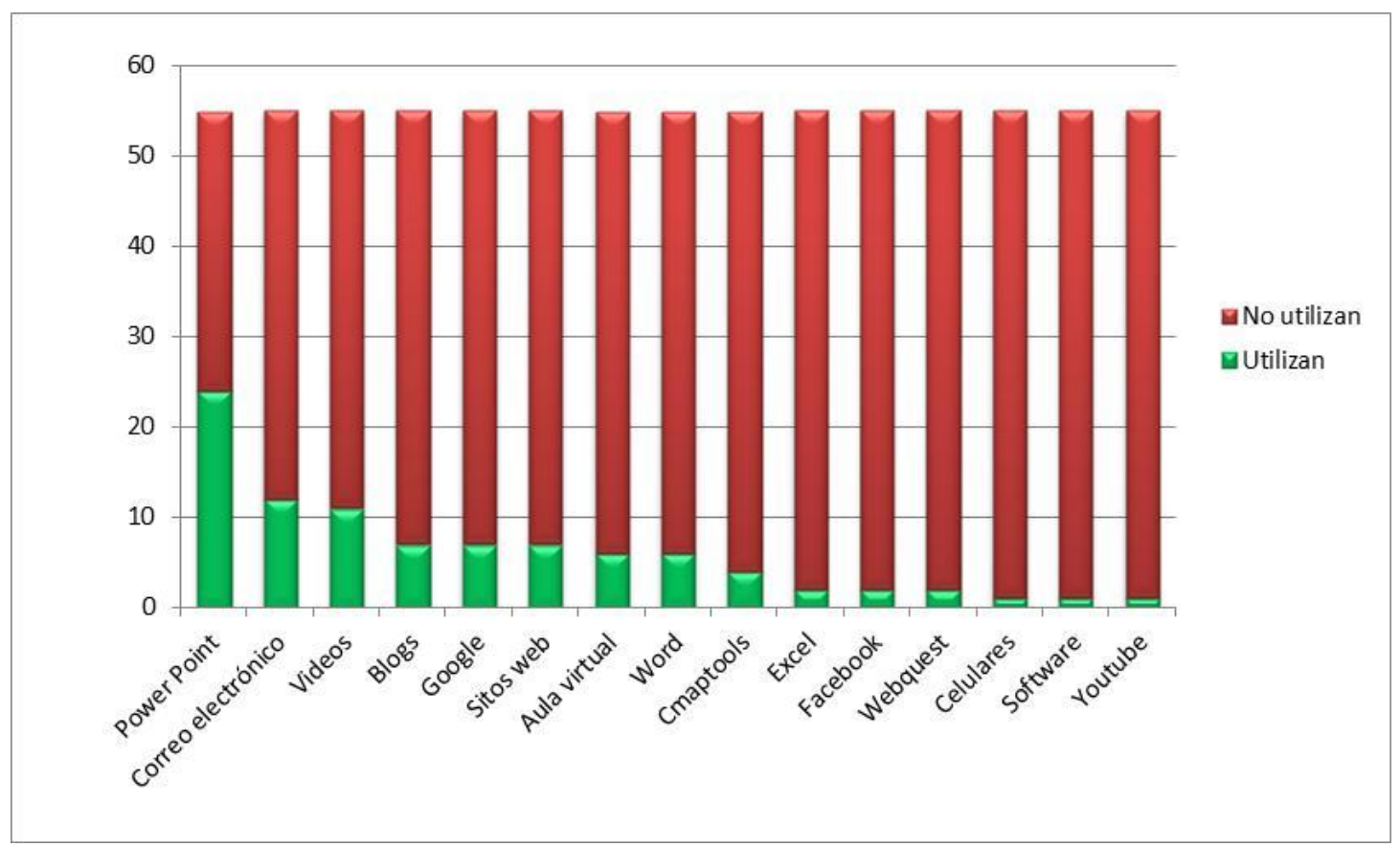

Figura 1. Herramientas utilizadas por los encuestados

Durante la realización del seminario se registró la participación y desgranamiento vinculado a cada actividad y/o contenido obteniéndose los resultados que se muestran en la Figura 2. Como puede observarse, la participación de los inscriptos fue mermando en el transcurso de las 8 semanas, realizando el trabajo final sólo un $12 \%$. Sin embargo un elevado número de inscriptos que no participó de las actividades, sí ingresó a la plataforma para descargar todo el material proporcionado.

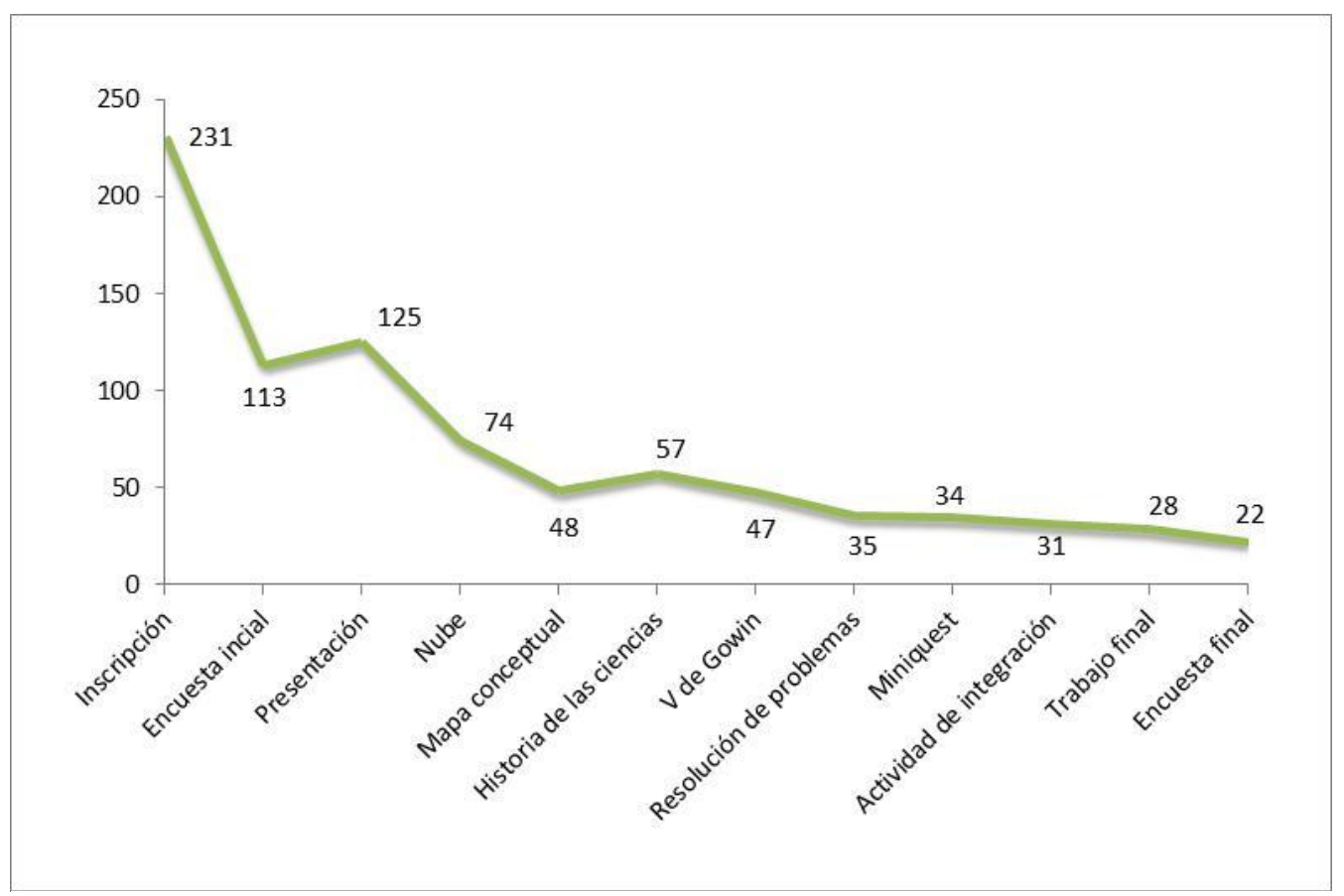

Figura 2. Número de profesores que cumplieron con cada una de las actividades propuestas 
Memorias del VII Encuentro Nacional de Experiencias en la Enseñanza de la Biologia y la Educación Ambiental y II Congreso Nacional de Investigación en la Enseñanza de la Biología

Los participantes tuvieron numerosas dificultades para realizar las actividades, a continuación haremos mención a algunas de ellas:

Mapas conceptuales: buena parte de los cursantes elaboraron "esquemas" que lejos estaban de ser mapas conceptuales eran oraciones dispuestas verticalmente, donde no aparecían ni los conceptos ni los conectores y menos aún una distribución jerárquica. Muchos, no respetaron la consigna de utilizar el Cmaptools para elaborar el mapa a partir de un tema concreto, el CDC, en su lugar subieron a la plataforma esquemas de los más diversos temas: evolución, metabolismo, función lineal, entre muchos otros.

Resolución de problemas: buena parte de los ejemplos propuestos por los colegas no fueron problemas pero tampoco ejercicios. Los profesores presentaron enunciados extremadamente largos con una sobreabundancia de información que dificultaba la comprensión y donde no quedaba claro que debían resolver concretamente.

V de Gowin: era desconocida por gran parte de los profesores, esto provocó dificultades para diferenciar, por ejemplo, las aseveraciones de valor de las de conocimiento, filosofía de teorías y principios. En tal sentido, debieron reelaborar las $\mathrm{V}$ en numerosas ocasiones y aun así no lograron aprobar la actividad.

Actividad de integración: presentó numerosas dificultades ya que debían resolver un problema concreto sobre fotosíntesis mediante la $\mathrm{V}$ de Gowin desarrollando los principios mediante un mapa conceptual.

\section{Conclusiones}

Al finalizar el seminario, realizamos una autoevaluación concluyendo que se lograron los objetivos que nos propusimos cuando fuimos convocadas para la capacitación.

Por otra parte, tanto los recursos como las herramientas contaron con la aceptación de los cursantes, ambos fueron calificados por la gran mayoría como "excelentes". En relación con los contenidos que se volcaron en cada una de las clases fueron valorados por su claridad, pertinencia, calidad, actualidad, otros.

Más allá de conocer o no las estrategias los profesores presentaron similares dificultades en la elaboración de las actividades. En relación con los mapas conceptuales, el principal problema que tuvieron fue elaborar una síntesis del CDC que les permitiera jerarquizar y establecer relaciones entre los conceptos. Muchos confundieron mapas con redes conceptuales, algunos objetaron su 
Edición Extra-Ordinaria. ISSN 2027-1034 P. p 895 - 902

Memorias del VII Encuentro Nacional de Experiencias en la Enseñanza de la Biologia y la Educación Ambiental y II Congreso Nacional de Investigación en la Enseñanza de la Biología

utilización en el aula y hasta hubo quienes insistieron en calificarlos como "herramientas" inventadas para transmitir y ordenar el conocimiento.

Por otra parte, los profesores tuvieron serias dificultades para proponer y resolver problemas y buena parte de ellos los confundieron con los ejercicios, situación muy generalizada en el colectivo docente. En cuanto a la Historia de la Ciencia debemos destacar varias cuestiones importantes, en principio el reconocimiento por parte de los cursantes que son los propios docentes los que se niegan a utilizarla. Las razones que esgrimieron fueron: ..hablar de Historia de la Biología es una verdadera pérdida de tiempo;... a los alumnos les resulta "aburridor" ponerse a leer toda la hora de Historia de la Biología, ...me gustaría saber cómo usarla sin tener que presentar un montón de fechas y nombres de manera que los alumnos no se aburran y se interesen.... Estas declaraciones muestran que para los profesores se trata de presentar una sucesión de fechas y nombres de científicos así como de disponer de largas horas de lectura, lo que sin lugar a dudas resulta tedioso para los alumnos y para el profesor.

La V de Gowin fue valorada como una estrategia "interesante" para ser utilizada en investigaciones y trabajos prácticos con los alumnos ya que permite establecer relaciones entre la teoría y la práctica. No obstante, el desconocimiento por parte de los profesores y la falta de experiencia en su implementación, sumada a la relativa complejidad que involucra su elaboración, no nos permitiría asegurar que la misma sea tenida en cuenta a la hora de planificar sus unidades didácticas.

Finalmente, y tal como ocurre la mayoría de las veces en que se pretende incorporar una estrategia a las clases nos encontramos con los trillados argumentos de: el elevado número de alumnos en las aulas, la carga horaria de las materias que es escasa y la cantidad de contenidos que se pretende que se enseñe no deja margen para incorporar ni los mapas, ni los problemas y mucho menos la historia de la ciencia, entre muchos otros argumentos.

El seguimiento de los cursantes en la plataforma se realizó de manera efectiva por parte de los tutores y los coordinadores. Esto permitió obtener valiosa información en relación con las fortalezas y debilidades del seminario. Entre las primeras destacamos la acertada selección de los contenidos, las herramientas, los portales y las actividades para una primera experiencia de este tipo. Entre las segundas mencionaremos: 1. La falta de tiempo para realizar las actividades. Los cursantes manifestaron que al menos hubieran necesitado dos semanas más. Muchas de las actividades debían ser subidas a la plataforma en fechas que coincidían con obligaciones escolares como cierre de promedios, evaluaciones, otras, lo que provocó que no pudieran dedicarse en profundidad a todas las clases. 2. La falta de experticia de muchos de los profesores para el manejo de los programas. Gran parte de ellos apenas utilizaban el correo electrónico esto provocó una serie de dificultades para bajar e instalar los software y para subir y comentar las actividades en los foros correspondientes. 
Bio-grafía Escritos sobre la Biologia y su Enseñanza.

Edición Extra-Ordinaria. ISSN 2027-1034 P.p 895 - 902

Memorias del VII Encuentro Nacional de Experiencias en la Enseñanza de la Biología y la Educación Ambiental y II Congreso Nacional de Investigación en la Enseñanza de la Biología

Por nuestra parte consideramos que el elevado desgranamiento observado entre los cursantes se debió a que el seminario no otorgaba puntaje y éste es relevante para ingresar al sistema educativo, ya que los méritos del profesor (título, capacitaciones, cursos, jornadas, otros) le otorga un puntaje y un orden de mérito en las materias y escuelas en que se inscribe todos los años.

\section{Bibliografía}

Choque, R. (2008): La integración de las TIC en el sistema educativo. Signo Educativo, 17(168), 36-39.

Cmaptools. En: http://cmap.ihmc.us/conceptmap.html

Decreto 459 (2010). Boletín Oficial del 07/04/10. Educación. Crea Programa Conectar la Igualdad.com.ar de incorporación de las nuevas tecnologías para el aprendizaje de alumnos y docentes. En: www.conectarigualdad.gob.ar

History.com. En: http://www.tuhistory.com.

Jing Project. En: http://jingproject.uptodown.com/.

Portal Educativo de Chile. http://www.educarchile.cl/

Sánchez, J. (2002). Integración curricular de las TICs. Conceptos e ideas. Ponencia presentada en VI Congreso lberoamericano de informática educativa. RIBIE, Vigo, España.

Webquest. En: http://www.aula21.net/tercera/estructura.htm http://coleccion.educ.ar/coleccion/CD4/contenidos/ ..http://www.webquest.es/estructura-de-la-webquest

Wordle. En: http://www.wordle.net/create 